in those directions or the interference from a known angular region can be reduced by lowering the sidelobe levels in that angular region only. The technique encompasses all aperture surfaces whose integral equations can be solved either classically or numerically.

\section{REFERENCES}

[1] T. T. Taylor, "Design of line source antennas for narrow beamwidth and low sidelobes," IRE Trans. Antennas Propagat., vol. AP-3, pp. 16-28, Jan. 1955.

[2] A. R. Lopez, "A line source excitation for maximum aperture efficiency with a given sidelobe level," presented at the 1970 G-AP Int. Symp. Sept. 14-16, 1970.

[3] E. J. Powers, "Utilization of the Lamda functions in the analysis and synthesis of monopulse antenna difference patterns," IEEE Trans. Antennas Propagat., vol. AP-15, pp. 771-777, Nov. 1967.

[4] E. T. Bayliss, "Design of monopulse antenna difference patterns with low sidelobes," Bell Syst. Tech. J., vol. 47, pp. 623-6.50, May-June 1968 .

[5] R. F. Hyneman, "A technique for the synthesis of line source antenna patterns having specified sidelobe behaviour," IEEE Trans. Antennas Propagat., vol. AP-16. pp. 430-435, July 1968.
[6] R. R. Kinsey, "Line source aperture monopulse excitations," General Electric Co., AFCRL-68-0254, Apr. 1968.

[7] S. M. Sanzgiri and J. K. Butler, "Constrained optimization of the performance indices of arbitrary array antennas," IEEE Trans. Aniennas Propagal., vol. AP-19, pp. 493-498, July 1971.

[8] D. R. Rhodes, "The optimum line source for the best meansquare approximation to a radiation pattern," IEEE Trans. Antennas Propagat., vol. AP-11, pp. 440-446, July 1963.

[9] R. R. Kinsey, "Monopulse difference slope and gain standards," IRE Trans. Antennas Propagat., vol. AP-10, pp. 343-344, May 1962.

[10] D. Slepian and H. O. Pollack, "Prolate spheroidal wave functions, Fourier analysis and uncertainty-I," Bell Syst. Tech. $J$., vol. 40, pp. $65-84$, Jan. 1961.

[11] D. R. Rhodes, "On some double orthogonality properties of the spheroidal and Mathieu functions," J. Math. Phys., vol. 44, pp. 52-65, Mar. 196\%.

[12] I). Slepian. "Prolate spheroidal wave functions, Fourier analysis and uncertainty-IV: Extensions to many dimensions; generalized prolate spheroidal functions," Bell Syst. Tech. J., vol. 43, pp. 3009-30-57, Nov. 1964.

[13] J. C. Helurtley, "Hyperspheroidal functions-optical resonators with circular mirrors," presented at Symp. Quasi-Optics, Polytech. Inst. Brooklyn, Brooklyn, N. Y., June 1964.

[14] J. A. Morrison, "Eigenfunctions of the finite Fourier transform operator over a hyperellipsoidal region," J. Math. Phys., vol. 44, pp. $245-2.54$, Sept. 1965.

\title{
Dipole Antenna in Space-Time Periodic Media
}

\author{
CHARLES ELACHI, MEMBER, IEEE
}

\begin{abstract}
The problem of dipole radiation in sinusoidally spacetime periodic media is studied and solved. The space-time periodicity can be considered as due to a strong pump wave and is expressed as a traveling-wave type change in the dielectric constant or the plasma density (i.e., $\epsilon(z, t)=\epsilon_{0} \epsilon_{r}\left[1+\epsilon_{1} \cos (K z-\Omega t)\right]$ and $N(z, t)=$ $\left.N_{0}\left[1+N_{1} \cos (K z-\Omega t)\right]\right)$. The solution also covers the limit case of a sinusoidally stratified medium $(\Omega=0)$. The solution is formulated in a matrix form, such that the basic results and diagrams apply, with minor changes, to the different cases studied: electric and magnetic dipole in a dielectric, plasma, and uniaxial plasma. The wave-vector diagram is used extensively in studying and presenting the different properties of the solution: caustics, effect of the disturbance (pump wave) motion, harmonics, radiation outside the allowed cone in a uniaxial plasma. Many dipole radiation patterns are given and their features explained physically. Finally, our solution and results are extended to the generally space-time periodic media where $\epsilon(z, t)$ and $N(z, t)$ behave as $\eta(z, t)=\eta_{0}[1+$ $\left.\eta_{1} f(K z-\Omega t)\right]$ where $f(\xi)$ is any periodic function.
\end{abstract}

\section{INTRODUCTION}

$\mathbf{T}$ THE STUDY of electromagnetic waves in space-time periodic media has occupied the attention of several researchers in recent years. The space-time periodicity

Manuseript received August 25, 1971; revised November 30 , 1971. This work was supported by NASA under Contract NAS 7-100 and by the California Institute of Technology under the Air Force Grant AFOSR-68-1400.

The author is with the Jet Propulsion Laboratory, California Institute of Technology, Pasadena, Calif. 91109. can be considered due to a strong pump wave (of the electromagnetic, acoustic, or other type) which modulates the permittivity of a dielectric or the electron density of a plasma in a propagating wave-like manner (i.e. $\eta(z, t)=\eta_{0}\left[1+\eta_{1} f(K z-\Omega t)\right]$ where $f(\xi)$ is a periodic function). Propagation of TE waves in infinite and bounded space-time periodic dielectric was studied by Simon [1], Cassedy and Oliner [2], Rao [3] and others. Askne [4] studied the TE case in an isotropic cold plasma and Elachi [5], [6] extended the study to both polarizations (TE and TMI) in different media (isotropic and uniaxial dielectric and plasma).

Waves excited by sources within the medium were only studied in the case of space-periodic media $(\Omega=0)$. The dipole radiation in a periodically stratified dielectric was studied by Casey [7] and the line source by Singer and Tamir [8]. Other workers investigated the radiation from a uniformly moving charged particle in a dielectric [9] and an isotropic plasma [10].

In this paper, we give the formal solution for the field and the radiation patterns of a dipole (electric and magnetic) in different sinusoidally space-time periodic media (dielectric, isotropic, and uniaxial plasma) and we investigate the special features: effect of the motion of the modulation, caustics, radiation outside the propagation cone in a uniaxial plasma. Finally our study is extended to generally space-time periodic media where $\epsilon(z, t)$ 
and $N(z, t)$ behave like $\eta(z, t)=\eta_{0}\left[1+\eta_{1} f(K z-\Omega t)\right]$ where $f(\xi)$ is any periodic function. This study also covers, as a special case $(\Omega=0)$, the radiation in a periodically stratified medium. In another paper, we study the case where the source is a uniformly moving charge.

\section{Solution and Patrerins of Radiated Field in Dielectric}

We consider a dipole parallel to the $z$ axis radiating in a dielectric with

$$
\epsilon(z, t)=\epsilon_{0} \epsilon_{r}\left[1+\epsilon_{1} \cos (K z-\Omega t)\right]
$$

where $(K, \Omega)$ are the wavenumber and angular frequency of the dielectric fluctuation. The geometry of the problem is shown in Fig. 1. Some notations are given in Table I.

\section{A. Magnetic Dipole}

The magnetic dipole will radiate a wave with an electric field parallel to the fluctuation wavefront $\left(E=E(\rho, z, t) e_{\phi}\right)$ and the corresponding wave equation outside the source is

$$
\left(\frac{\partial^{2}}{\partial \rho^{2}}+\frac{1}{\rho} \frac{\partial}{\partial \rho}-\frac{1}{\rho^{2}}+\frac{\partial^{2}}{\partial z^{2}}\right) E-\mu_{0} \frac{\partial^{2} \epsilon E}{\partial t^{2}}=0 .
$$

Using the separation of variables method and Floquet's theorem, we can write an elementary solution

$$
E^{(e)}=\sum_{n=-\infty}^{n=+\infty} E_{n} B_{1}(\delta \rho) \exp [i(\kappa+n K) z-i(\omega+n \Omega) t] d \kappa
$$

where

$$
B_{1}= \begin{cases}J_{1}, & \text { Bessel function for } \rho<a \\ H_{1}^{(1)}, & \text { Hankel function for } \rho>a\end{cases}
$$

and $a$ is the radius of the magnetic dipole loop. The expression of the field is determined by integrating over all the values of $\kappa$ or $\delta$ ( $\kappa$ and $\delta$ are related by the dispersion relation) with an adequate weighting function, thus the electric field is given by

$$
\begin{aligned}
E=\int_{-\infty}^{+\infty} W(\kappa) & \sum_{n} E_{n} B_{1}(\delta \rho) \\
\cdot & \cdot \exp [i(\kappa+n K) z-i(\omega+n \Omega) t] d \kappa .
\end{aligned}
$$

Putting the expression of $E$ into the wave equation and equating the terms with the same frequency we get the infinite system of equations

$$
D_{n} E_{n}+E_{n+1}+E_{n-1}=0
$$

or in a matrix form:

$$
\|M\| \cdot|E|=0
$$

where $|E|$ is an infinite column vector of elements $E_{n}$, $\|M\|$ is an infinite tridiagonal matrix with elements given in (4), and $D_{n}$ is given in Table I. As we are

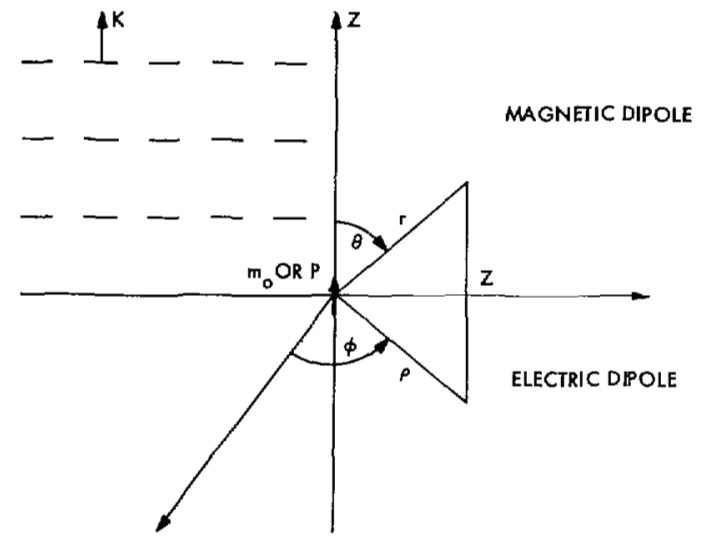

Fig. 1. Dipole in space-time periodic medium. Magnetic dipole: $E=E e_{\phi}, H=H_{p} e_{\rho}+H_{z} e_{z-}$ Electric dipole: $H=H e_{\phi}, E=$ $E_{\rho} e_{\rho}+E_{z} e_{z}$.

looking for a nontrivial solution, then we must have

$$
\Delta(\delta, \kappa, \omega)=\operatorname{det}\|M\|=0 .
$$

This is the dispersion relation which gives $\delta(\kappa)$ and the wave-vector diagram; these were studied in detail by the author in other papers [5], [6]. An example of the wave-vector diagram is given in Fig. 2(a) which shows that the solution is multivalued and, using the superposition principle, the field has to be written

$$
\begin{aligned}
E=\boldsymbol{e}_{\phi} \sum_{q=-\infty}^{q=+\infty} \sum_{n=-\infty}^{n=+\infty} \int_{-\infty}^{+\infty} W_{q}(\kappa) E_{n q}(\kappa) B_{1}\left(\delta_{q} \rho\right) \\
\cdot \exp \left[i(\kappa+n K) z-i \omega_{n} t\right]
\end{aligned}
$$

where $\delta_{q}(\kappa)$ are the different values of $\delta$, and $E_{n q}$ corresponds to the amplitude of the $n$th harmonic of the $q$ th mode. The relative values $C_{n q}=E_{n q} / E_{0 q}$ can be determined by solving (5) or from (4) [2], [5], [6]. Taking $\alpha_{q}(\kappa)=W_{q}(\kappa) E_{0 q}(\kappa) / \delta_{q}$, the field expression becomes

$$
\begin{aligned}
E=e_{\phi} \sum_{q=-\infty}^{q=+\infty} \sum_{n=-\infty}^{n=+\infty} \int_{-\infty}^{+\infty} & \alpha_{q} \delta_{q} C_{n q} B_{1}\left(\delta_{q} \rho\right) \\
& \cdot \exp \left[i(\kappa+n K) z-i \omega_{n} t\right] d \kappa .
\end{aligned}
$$

The magnetic field is determined from Maxwell's equations as

$$
\begin{array}{r}
H_{p}=-\frac{1}{\mu_{0}} \sum_{q} \sum_{n} \exp \left(-i \omega_{n} t\right) \int_{-\infty}^{+\infty} \frac{\kappa+n K}{\omega_{n}} \alpha_{q} \delta_{q} C_{n q} B_{1}\left(\delta_{q \rho}\right) \\
\cdot \exp [i(\kappa+n K) z] d \kappa
\end{array}
$$

$H_{z}=\frac{i}{\mu_{0}} \sum_{q} \sum_{n} \exp \left(-i \omega_{n} t\right) \int_{-\infty}^{+\infty} \frac{\delta_{q}}{\omega_{n}} \alpha_{q} \delta_{q} C_{n q} B_{0}\left(\delta_{q \rho}\right)$

$$
\cdot \exp [i(\kappa+n K) z] d \kappa \text {. }
$$

For $\rho<a$, we replace $\alpha_{q}$ by $\alpha_{q}^{\prime}$ because the weighting functions are not the same inside and outside the loop. Satisfying the source conditions:

$$
\left.E_{\phi}\right|_{g \rightarrow a} \text { is continuous } \rightarrow \alpha_{q} H_{1}^{(1)}\left(\delta_{q} a\right)=\alpha_{q}^{\prime} J_{1}\left(\delta_{q} a\right)
$$


TABLE I

Expressions of Parameters

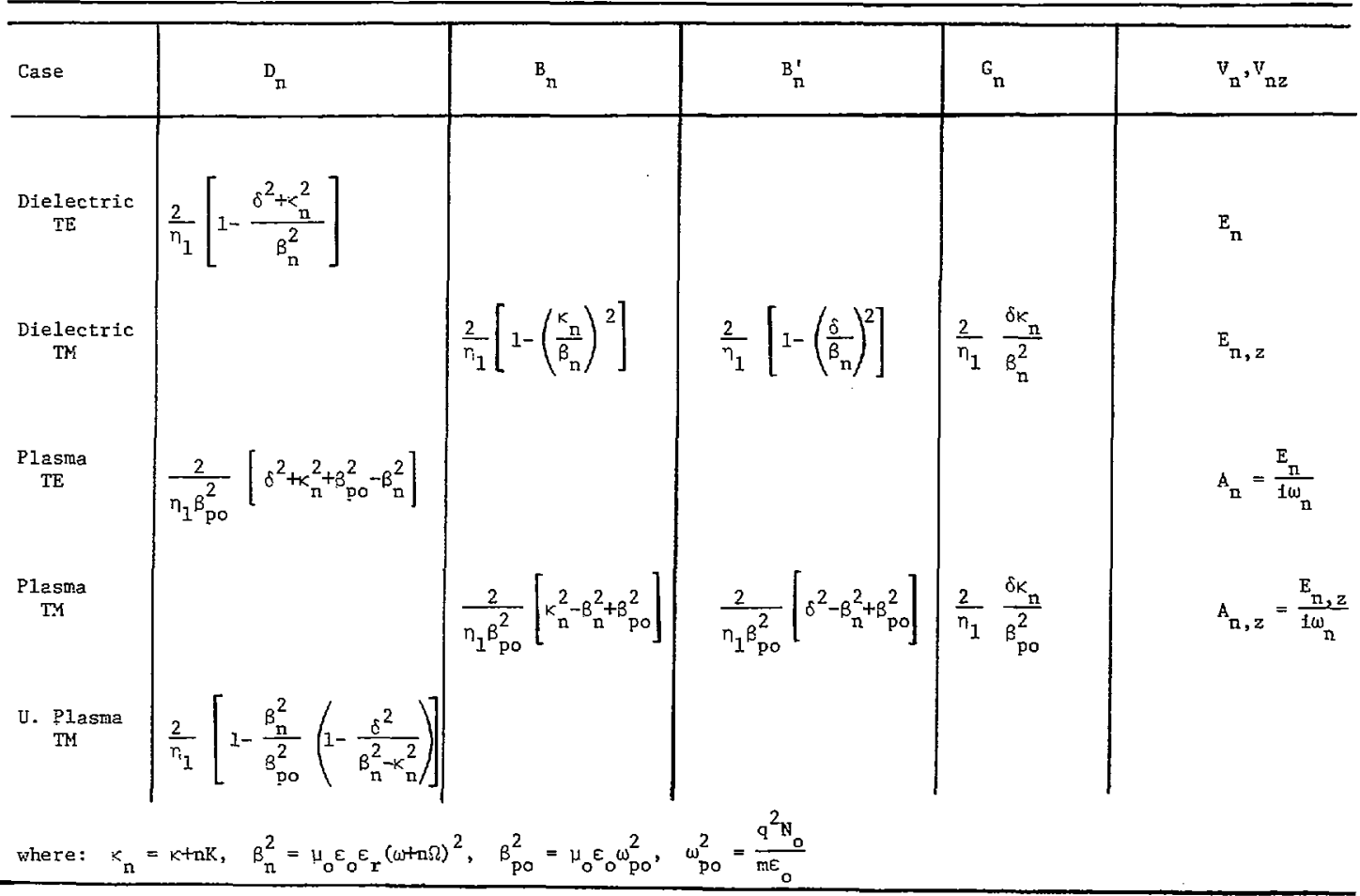

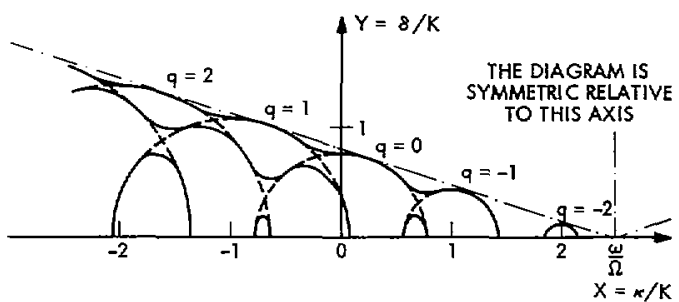

(a)

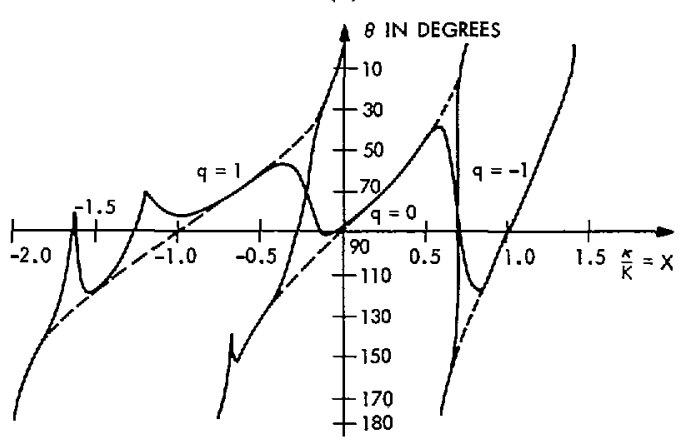

(b)

Fig. 2. (a) wave-vector diagram. (b) $\theta$ function of $K_{s} / K$ for sinusoidally space-time periodic dielectric: $R=V_{c} / V_{d}=3.5$, $\omega / \Omega=2.5, \quad \epsilon_{1}=0.25$ (dashed line corresponds to $\epsilon_{1} \rightarrow 0$ ). Envelope slope is $\simeq\left(R^{2}-1\right)^{-1 / 2}$. and Ampere's law:

$\left[H_{z}(\rho>a)-H_{z}(\rho<a)\right]_{\rho \rightarrow a}=I_{0} \delta(z) \exp (-i \omega t)$

where $\left(I_{0}, \omega\right)$ are the amplitude and frequency of the loop's current, we get

$\sum_{q} \sum_{n} \int_{-\infty}^{+\infty} \frac{\alpha_{q} \delta_{q} C_{n q}}{J_{1}\left(\delta_{q} a\right)} \exp \left[i(\kappa+n K) z-i \omega_{n} t\right] d \kappa$

$$
=\frac{\pi}{2} \mu_{0} a I_{0} \delta(z) \exp (-i \omega t) .
$$

Taking the limit $a \rightarrow 0$ and writing $\delta(z)$ in an integral form

$$
\delta(z)=\int_{-\infty}^{+\infty} \exp (i \kappa z) d \kappa
$$

we find

$$
\begin{aligned}
\int_{-\infty}^{+\infty}\left[\sum_{q} \sum_{n} \alpha_{q} C_{n q} \exp [i n(K z-\Omega t)]+\frac{\mu_{0} m_{0}}{4}\right] \\
\cdot \exp [i(\kappa z-\omega t)] d \kappa=0
\end{aligned}
$$

where $m_{0}=\pi a^{2} I_{0}=$ magnetic dipole moment. This relation must be true for all values of $z$ and $t$, therefore, we must have

$$
\begin{aligned}
& \sum_{q} C_{n q} \alpha_{q}=0, \quad \text { for } n \neq 0 \\
& \sum_{q} C_{0 q} \alpha_{q}=-\frac{\mu_{0} m_{0}}{4}
\end{aligned}
$$


this gives in a matrix form

$$
\|C\| \cdot|\alpha|=|M| \rightarrow|\alpha|=\|C\|^{-1} \cdot|M|
$$

where

$\|C\|=$ infinite matrix with elements $C_{n q}$

$|\alpha|=$ infinite column vector with elements $\alpha_{q}$

$|M|=$ column vector with elements $\left\{\begin{array}{r}M_{n}=0 \\ \text { for } n \neq 0 \\ M_{n}=-\frac{\mu_{0} m_{0}}{4} \\ \quad \text { for } n=0 .\end{array}\right.$

Therefore, the field expression is completely determined by

$$
\begin{array}{r}
E_{\rho>\alpha}=\sum_{q} \sum_{n} \exp \left(-i \omega_{n} t\right) \int_{-\infty}^{+\infty} \alpha_{q}(\kappa) \delta_{q}(\kappa) C_{n q}(\kappa) H_{1}{ }^{(1)}\left(\delta_{q} \rho\right) \\
\cdot \exp [i(\kappa+n K) z] d \kappa . \quad(15)
\end{array}
$$

The integral will be evaluated in the far field by the steepest-descent method.

\section{B. Electric Dipole}

The EM field radiated by an electric dipole is TM relative to the $z$ axis (i.e., $H=H e_{\phi}, E=E_{\rho} e_{p}+E_{z} e_{z}$ ). Applying Floquet's theorem directly to Maxwell's equations and following the same method used for the magnetic dipole, we can write

$$
\begin{aligned}
H=e_{\phi} \sum_{q} \sum_{n} \int_{-\infty}^{+\infty} W_{q}(\kappa) H_{n q}(\kappa) H_{1}^{(1)}\left(\delta_{q} \rho\right) \\
\cdot \exp \left[i(\kappa+n K) z-i \omega_{n} t\right] d \kappa
\end{aligned}
$$

$$
\begin{array}{r}
E=\sum_{q} \sum_{n} \int_{-\infty}^{+\infty} W_{q}\left[-i E_{n q z} H_{0}^{(1)}\left(\delta_{q} \rho\right) \boldsymbol{e}_{z}+E_{n q \rho} H_{1}{ }^{(1)}\left(\delta_{q} \rho\right) \boldsymbol{e}_{\rho}\right] \\
\cdot \exp \left[i(\kappa+n K) z-i \omega_{n} t\right] d \kappa . \quad(17)
\end{array}
$$

Putting these expressions in Maxwell's equations and equating the terms with the same frequency we get (the index $q$ is disregarded for notation clarity)

$$
\begin{aligned}
& H_{n}=-\frac{\epsilon_{0} \epsilon_{r} \omega}{\delta}\left(E_{n z}+\frac{\epsilon_{1}}{2} E_{n+1, z}+\frac{\epsilon_{1}}{2} E_{n-1, z}\right) \\
& E_{n \rho}=-\frac{1}{G_{n}}\left(B_{n}{ }^{\prime} E_{n z}+E_{n+1, z}+E_{n-1, z}\right)
\end{aligned}
$$

and the infinite system of equations

$$
\begin{array}{r}
{\left[\frac{B_{n} B_{n}^{\prime}-G_{n}^{2}}{G_{n}}+\frac{1}{G_{n+1}}+\frac{1}{G_{n-1}}\right] E_{n z}+\left[\frac{B_{n}}{G_{n}}+\frac{B_{n+1} 1^{\prime}}{G_{n+1}}\right] E_{n+1, z}} \\
+\left[\frac{B_{n}}{G_{n}}+\frac{B_{n-1}{ }^{\prime}}{G_{n-1}}\right] E_{n-1, z}+\frac{1}{G_{n+1}} E_{n+2, z}+\frac{1}{G_{n-1}} E_{n-2, z}=0
\end{array}
$$

or in a matrix form: $\|M\| \cdot\left|E_{z}\right|=0$, where $B_{n}, B_{n}{ }^{\prime}$, $G_{n}$ are given in Table $\mathrm{I}$ and $\|M\|=$ infinite matrix with elements given by (20) and $\left|E_{z}\right|=$ infinite column vector with elements $E_{n z}$. The dispersion equation is

$\Delta(\delta, \kappa, \omega)=\operatorname{det}\|M\|=0$ (nontriviality condition).

This equation has multiple solutions $\delta_{q}(\kappa)[5],[6]$ and using the superposition principle, we have to sum overall the indexes $q$ (this explains the summation over $q$ in (16) and (17)).

The relative amplitudes: $C_{n q}^{\prime}=E_{n q z} / E_{0 q z}, \quad F_{n q}^{\prime}=$ $E_{n q \rho} / E_{0 q z}$ and $A_{n q}{ }^{\prime}=H_{n q} / E_{0 q z}$ are determined from (18) $-(20)$ [5].

Taking $\alpha_{q}=W_{q} E_{0 q z} / \delta_{q}$, the field expression becomes

$$
\begin{aligned}
H=e_{\phi} \sum_{q} \sum_{n} \int_{-\infty}^{+\infty} \alpha_{q} A_{n q}{ }^{\prime} \delta_{q} H_{1}^{(1)}\left(\delta_{q} \rho\right) \\
\cdot \exp \left[i(\kappa+n K) z-i \omega_{n} t\right] d \kappa .
\end{aligned}
$$

The weighting functions $\alpha_{q}$ are determined from the source condition

$$
\lim _{\rho \rightarrow 0} 2 \pi \rho H_{\phi}=I=-i \omega p \delta(z) \exp (-i \omega t)
$$

where $p$ is the electric dipole moment. This condition gives, after writing $\delta(z)$ in an integral form,

$$
\begin{aligned}
\int_{-\infty}^{+\infty}\left[\sum_{q} \sum_{n} \alpha_{q} A_{n q}^{\prime} \exp [i n(K z-\Omega t)]-\frac{\omega p}{4}\right] \\
\cdot \exp [i(\kappa z-\omega t)] d \kappa=0 .
\end{aligned}
$$

This relation will be satisfied for all $z$ and $t$ if

$$
\begin{aligned}
& \sum_{q} \alpha_{q} A_{n q}{ }^{\prime}=0, \quad \text { for } n \neq 0 \\
& \sum_{q} \alpha_{q} A_{0 q}{ }^{\prime}=\frac{\omega}{4} p
\end{aligned}
$$

which gives, in a matrix form,

$$
\left\|A^{\prime}|| \cdot|\alpha|=|P| \rightarrow|\alpha|=\right\| A^{\prime}||^{-1} \cdot|P|
$$

where $\left\|A^{\prime}\right\|$ is a matrix with elements $A_{n q}{ }^{\prime}$ and $|P|$ is a column vector with elements

$$
P_{0}=\frac{\omega}{4} p \quad \text { and } \quad P_{n}=0, \quad \text { for } n \neq 0
$$

Having $|\alpha|$, the field is completely determined by (21). In the far field the integral can be determined with the steepest-descent method.

\section{Sonic Region}

Applying Poincare's convergence theorem to the system of (4), (20), we find that in the region

$$
\frac{V_{c}}{\left(1+\epsilon_{1}\right)^{1 / 2}} \leq \frac{\Omega}{K} \leq \frac{V_{c}}{\left(1-\epsilon_{1}\right)^{1 / 2}}
$$


where $V_{c}=\left(\mu_{0} \epsilon_{0} \epsilon_{r}\right)^{-1 / 2}$, the solution does not converge and there is a blow up of the harmonics [2], [6].

\section{Asymptotic Evaluation of Integral}

Only the magnetic dipole field is determined in this section. The same method, using the corresponding matrix $\|M\|$, can be used for the electric dipole field.

When $p \rightarrow \infty$, the electric-field expression from (15) becomes

$$
\begin{aligned}
E=-i\left(\frac{2}{\pi r \sin \theta}\right)^{1 / 2} \exp \left(-i \frac{\pi}{4}\right) & \sum_{q} \sum_{n} \int_{-\infty}^{\infty} \delta_{q}^{1 / 2} \alpha_{q} C_{n q} \\
& \cdot \exp \left[i r F_{q}(\kappa)-i \omega_{n} t\right] d \kappa
\end{aligned}
$$

where

$$
\begin{gathered}
F_{q}(\kappa)=(\kappa+n K) \cos \theta+\delta_{q} \sin \theta \\
r^{2}=\rho^{2}+z^{2}, \quad \tan \theta=\frac{\rho}{z}
\end{gathered}
$$

Using the steepest-descent method when $r \rightarrow \infty$, we find

$$
\begin{aligned}
E=- & \frac{2 i}{r \sin \theta} \sum_{j} \sum_{q} \sum_{n}\left(\frac{\delta_{q s}}{\delta_{q s}{ }^{\prime \prime} \mid}\right)^{1 / 2} \alpha_{q s} C_{n q s} \\
& \cdot \exp \left[-i \omega_{n} t+i r F_{q s}-i \frac{\pi}{4}\left(1-\operatorname{sign} \delta_{q s}{ }^{\prime \prime}\right)\right]
\end{aligned}
$$

where the index $s$ means value at the saddle point and the summation over $j$ means summation over all the saddle points (it is possible to have more than one). The saddle points are determined by

$$
\left.\frac{d F_{q}}{d \kappa}\right|_{\kappa=\kappa_{s}}=\left.0 \rightarrow \frac{d \delta_{q}}{d \kappa}\right|_{\delta_{q}=\delta_{q s, \kappa=\kappa_{s}}}=-\cot \theta .
$$

In Fig. 2(b) we give $\theta$ function of $X=\kappa_{s} / K$ which correspond to the wave vector diagram of Fig. 2(a). This curve gives, for each value of $\theta$ the corresponding values of $\kappa=\kappa_{s}$ and then we get $\delta_{q}=\delta_{q s}$ from the wavevector diagram.

\section{E. Caustics}

A very interesting effect appears when $\epsilon_{1} \neq 0$ which is the apparition of inflection points, near the interaction region between two modes (i.e. stopband), in the wave-vector diagram. At these points, $\delta^{\prime \prime}=0$ and the feld given by (22) is very large. This is a focalization and radiation enhancement effect, due to the inhomogeneity of the medium, on a certain surface called "caustic." In this problem the caustics are conical surfaces. The caustic cone angle is given by the slope of $\delta(\kappa)$ at the inflection point

$$
\cot \theta_{c}=-\left.\frac{d \delta}{d \kappa}\right|_{\text {at inflection point }}
$$

\section{F. Field Amplitude and Radiation Pattern}

Let us take $B_{n q}=\|C\|_{n q}{ }^{-1}$, where $\|C\|$ is the matrix with elements $C_{n q}$, then from (11), we get

$$
\alpha_{q}=-\frac{1}{4} \mu_{0} m_{0} B_{0 q}
$$

and the square of the $n$th harmonic amplitude is given by

$$
\begin{aligned}
\left|e_{n}\right|^{2}= & e_{n} e_{n}{ }^{*} \\
= & \left(\frac{\mu_{0} m_{0}}{2 r \sin \theta}\right)^{2}\left[\sum_{j} \sum_{q} \frac{\delta_{q s}}{\left|\delta_{q s}{ }^{\prime \prime}\right|}\left(B_{0 q} C_{n q}\right)^{2}\right. \\
& +\sum_{j} \sum_{q} \sum_{p \neq \neq j} \sum_{m \neq q}\left(\frac{\delta_{q s} \delta_{m s}}{\left|\delta_{q s}{ }^{\prime \prime} \delta_{m s}{ }^{\prime \prime}\right|}\right)^{1 / 2} B_{0 q} B_{0 m} C_{n q} C_{n m} \\
& \left.\cdot \exp \left[i r\left(F_{q s}-F_{m s}\right)\right]\right] .
\end{aligned}
$$

The second term is oscillatory and we can drop it if we average over the distance $r$, and the expression of the $n$th harmonic normalized average pattern becomes

$$
\begin{aligned}
G_{n}(\theta) & =\left[r^{2}\left|e_{n}\right|^{2}\right]_{\text {normalized }} \\
& =\frac{1}{\beta_{0}{ }^{2} \sin ^{2} \theta} \sum_{j} \sum_{q} \frac{\delta_{q s}}{\left|\delta_{q s}{ }^{\prime \prime}\right|}\left(B_{0 q} C_{n q}\right)^{2}
\end{aligned}
$$

For $\epsilon_{1} \rightarrow 0$, this pattern reduces to

$$
\begin{aligned}
& G_{n}(\theta)=0, \quad \text { for } n \neq 0 \\
& G_{0}(\theta)=\sin ^{2} \theta .
\end{aligned}
$$

In Figs. $3-5$ we give the patterns for a stationary disturbance $(\Omega=0)$ and the fundamental and 1st harmonic for a moving disturbance $(\Omega \neq 0)$ where

$\lambda=\frac{2 \pi}{\omega}\left(\mu_{0} \epsilon_{0} \epsilon_{r}\right)^{-1 / 2}, \quad \Lambda=\frac{2 \pi}{K}, \quad R=\frac{V_{c}}{V_{d}}=\frac{K}{\Omega}\left(\mu_{0} \epsilon_{0} \epsilon_{r}\right)^{-1 / 2}$.

The general features of these patterns are the following.

1) Stationary disturbance $(\Omega=0)$ :

For $\Lambda / \lambda=1.25$ (Fig. 3), there are four caustics at $\theta_{c}=43^{\circ}, 147^{\circ}, 82^{\circ}$, and $98^{\circ}$, and the pattern is symmetric relative to $\theta=90^{\circ}$.

For larger values of $\Lambda / \lambda$ more caustics appear and for smaller values we get less or no caustics. In fact, if $\lambda \gg \Lambda$, the EM wave will not see the disturbance and no focusing effect occurs. These results agree with Casey's results [7]

2) Moving disturbance $(\Omega \neq 0)$ :

The symmetry relative to $\theta=90^{\circ}$ is lost. The pattern and the caustic are pushed up by the motion of the disturbance. The fundamental mode $(q=0)$ contributes mostly to the fundamental harmonic $(n=0)$. The other modes contribute less except near a caustic. Similarly the mode $(q=m)$ contributes mostly to the harmonic $(n=m)$ except near a caustic where its contribution to the harmonics $(n=m \pm 1)$ is important. 


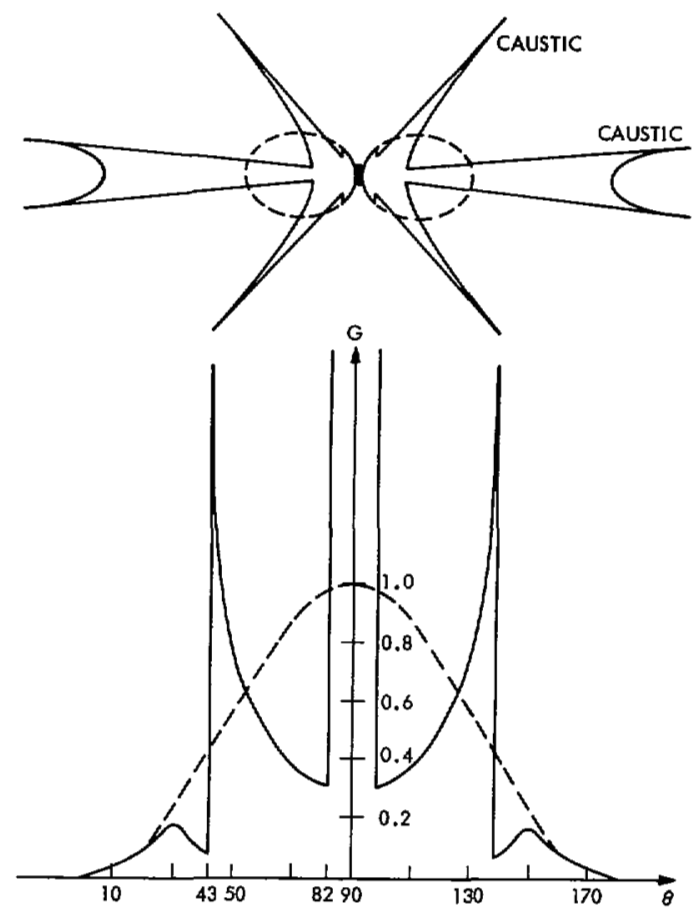

Fig. 3. Radiation pattern of dipole in sinusoidally stratified dielectric: $\lambda / \Lambda=0.8, \epsilon_{1}=0.25$ (dashed line corresponds to $\epsilon_{1} \rightarrow 0$ ).
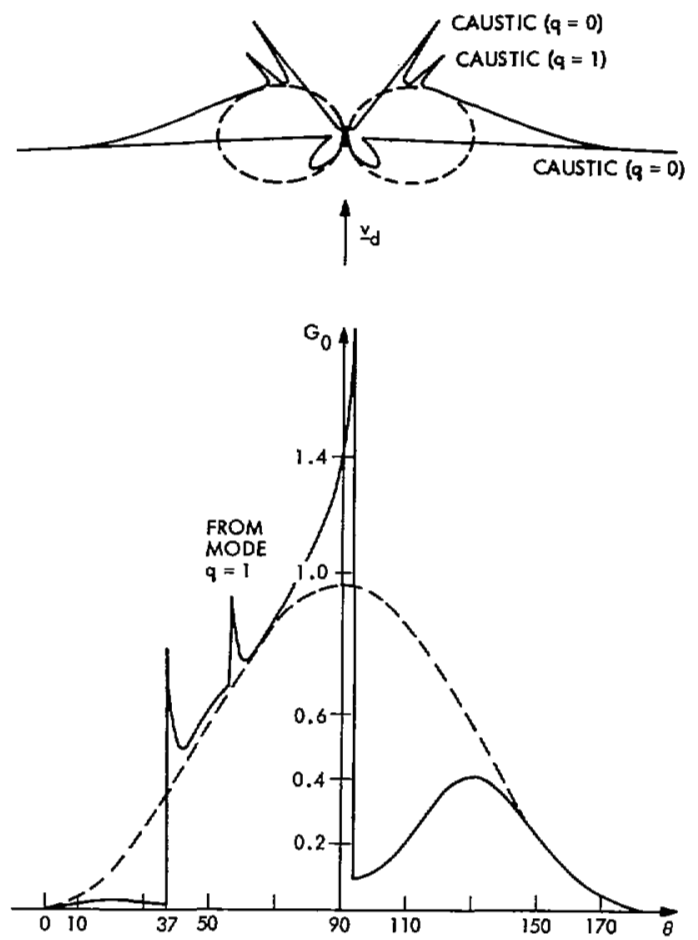

Fig. 4. Fundamental radiation pattern of dipole in sinusoidally space-time periodic dielectric: $\omega / \Omega=2.5, R=3.5, \epsilon_{1}=0.25$ (dashed line corresponds to $\epsilon_{1} \rightarrow 0$ ).
The acute asymetry in the figures is due to the highdisturbance velocity (i.e., $V_{d}=V_{c} / R$ ) and can be easily explained from the wave-vector diagram.

\section{Dipole Field in Plasma}

The wave equation for the magnetic dipole field is $[4],[5]$

$$
\nabla^{2} A-\mu_{0} \epsilon_{0} \frac{\partial^{2} A}{\partial t^{2}}-\mu_{0} \epsilon_{0} \omega_{p}^{2}(z, t) A=0
$$

where $E=\partial A / \partial t, A=A \boldsymbol{e}_{\phi}$, and

$$
\begin{aligned}
\omega_{p}^{2}(z, t) & =\frac{q^{2}}{m \epsilon_{0}} N(z, t)=\frac{q^{2} N_{0}}{m \epsilon_{0}}\left[1+N_{1} \cos (K z-\Omega t)\right] \\
\omega_{p 0}{ }^{2} & =\frac{q^{2} N_{0}}{m \epsilon_{0}} .
\end{aligned}
$$

The solution for $A$ has the form

$$
\begin{aligned}
A=\sum_{q=-\infty}^{q=+\infty} \sum_{n=-\infty}^{n=+\infty} \int_{-\infty}^{+\infty} W_{q}(\kappa) A_{n q} H_{I}^{(1)}\left(\delta_{q} \rho\right) \\
\cdot \exp \left[i(\kappa+n K) z-i \omega_{n} t\right] d \kappa .
\end{aligned}
$$

Putting the expression of $A$ in the wave equation and equating the terms of the same frequency, we get the infinite system of equations

$$
D_{n} A_{n}+A_{n+1}+A_{n-1}=0
$$

or

$$
\|M\| \cdot|A|=0
$$


where $D_{n}$ is given in Table I. The nontriviality condition

$$
\Delta(\delta, \kappa, \omega)=\operatorname{det}\|M\|=0
$$

gives the dispersion equation and the different solutions $\delta_{q}(\kappa)$. The relative amplitudes $C_{n q}=A_{n q} / A_{0 q}$ are determined from (26) or (27).

Using the previous method, we find

$E=e_{\phi} \sum_{q} \sum_{n} \int_{-\infty}^{+\infty} \alpha_{q} \delta_{q} C_{n q} \omega_{n} H_{1}^{(1)}\left(\delta_{q} \rho\right)$

$$
\cdot \exp \left[i(\kappa+n K) z-i \omega_{n} t\right] d \kappa
$$

where $|\alpha|=(i / \omega) \| C||^{-1} \cdot|m|$, and the normalized farfield pattern is

$$
G_{n}(\theta)=\frac{\omega_{n}^{2}}{\omega^{2}-\omega_{p 0}^{2}} \frac{1}{\beta_{0}^{2} \sin ^{2} \theta} \sum_{j} \sum_{q} \frac{\delta_{q s}}{\left|\delta_{q s}{ }^{\prime \prime}\right|}\left(B_{0 q} C_{n q}\right)^{2}
$$

with the same notations as the previous section. (For graphs of radiation patterns the reader is referred to [5].)

For $\omega+\Omega>\omega_{p 0}$, then even if the dipole frequency is lower than the plasma frequency, there is radiation of the high harmonics.

Finally, applying Poincaré's convergence theorem to (26), we find that there is no sonic region (divergence region) for a space-time periodic plasma [4], [5].

\section{Dipole in Uniaxial Plasma}

The dipole moment, the static field $B_{0}$ and the disturbance propagation vector are supposed to be parallel to the $z$ axis. With the condition $B_{0} \rightarrow \infty$, the wave equation is

$$
\nabla \times \nabla \times A+\mu_{0} \epsilon_{0} \frac{\partial^{2} A}{\partial t^{2}}+\mu_{0} \epsilon_{0} \omega_{p}^{2}(z, t) A \cdot e_{z}=0
$$

where $E=\partial A / \partial t$.

For a magnetic dipole, $E=E e_{\phi}$ (i.e., $E_{z}, A_{z}=0$ ), the field does not see the anisotropy and the problem is identical to a dipole in vacuum.

For the electric dipole, where $A=A_{z} e_{z}+A_{\rho} e_{\rho}$, we follow the same method used for a dielectric and we find $[5],[6]$

$$
D_{n} A_{n z}+A_{n+1, z}+A_{n-1, z}=0
$$

or

$$
\|M\| \cdot\left|A_{z}\right|=0
$$

where $D_{n}$ is given in Table $\mathrm{I}$. The dispersion relation is determined from the nontriviality condition and the pattern is determined in the same way used for the dielectric case [5].

In Figs. 6-8 we give the wave-vector diagram and the radiation pattern for the fundamental and $(\omega-\Omega)$ harmonic. The numerical values were chosen such that: $\omega>\omega_{p 0}$ and $\omega-\Omega<\omega_{p 0}$.

In a homogeneous uniaxial plasma $\left(N_{1}=0\right)$, it is well known that, for $\omega<\omega_{p 0}$, group propagation is possible only in a cone with axis parallel to $\boldsymbol{e}_{z}$ and half-

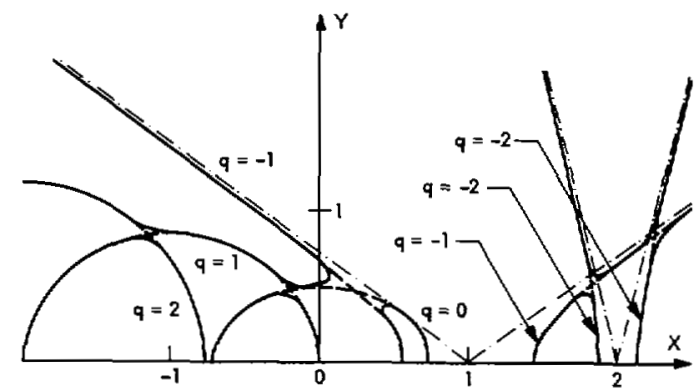

Fig. 6. Wave-vector diagram for sinusoidally space-time periodic uniaxial plasma: $R=3.5, \omega / \Omega=2.5, \omega_{p 0} / \omega=0.75, N_{1}=0.25$ (dashed line corresponds to $N_{1} \rightarrow 0$ ).
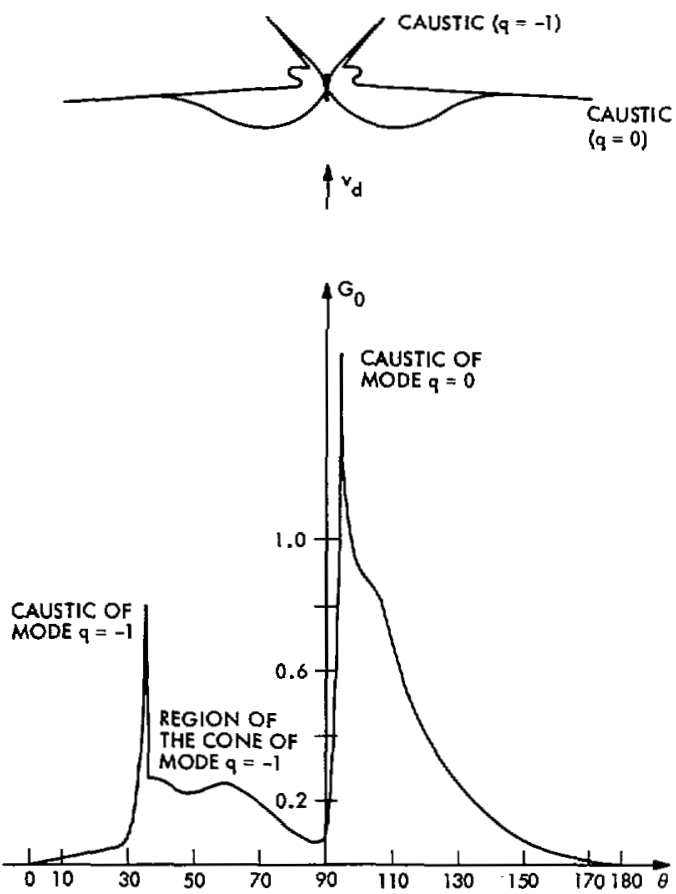

Fig. 7. Fundamental radiation pattern of dipole in sinusoidally space-time periodic uniaxial plasma: $R=3 . \overline{5}, \omega / \Omega=2.5$, $\omega_{p 0} / \omega=0.75, N_{1}=0.25$

angle $\theta=\arcsin \omega / \omega_{p 0}$, this can be seen from the corresponding wave-vector diagram which is a hyperbola with a slope

$$
\left(\frac{\omega_{p 0}^{2}}{\omega^{2}}-1\right)^{1 / 2} \leq \mid \text { slope } \mid<\infty
$$

the slope being related to the group angle $\theta_{g}$ by

$$
\cot \theta_{g}=\text { slope. }
$$

For the space-time periodic case $\left(N_{1} \neq 0\right)$, we see from Fig. 6 that for the hyperbolic modes, the slope goes to zero near the intersection points (stopbands) and this implies the possibility of radiation for all angles. This is clearly shown in the dipole pattern (Fig. 8) where the caustics and the cones are present. Radiation outside the cone also occurs in a stratified uniaxial plasma $(\Omega=0)[5]$, [6]. This effect can be explained easily from Fig. 9. Let us consider two phase rays, 1 and 2 , inside the phase cone angle. If at a point $A$ we take the vector sum of the rays 2 and $1^{\prime}$ (which is the reflected 


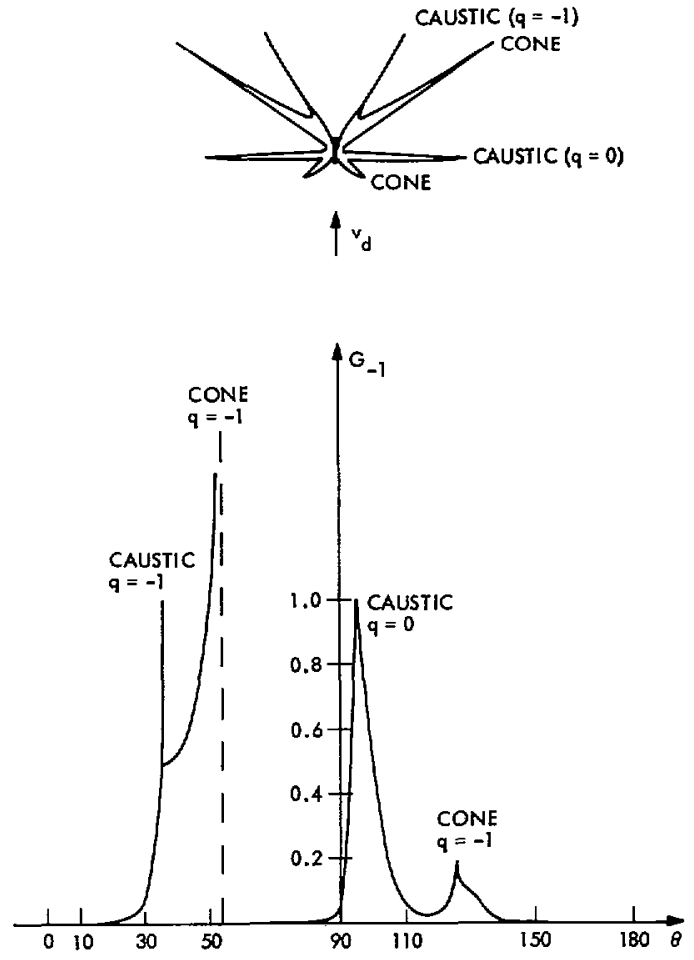

Fig. 8. Harmonic $(\omega-\Omega)$ pattern for same case as in Fig. 7 .

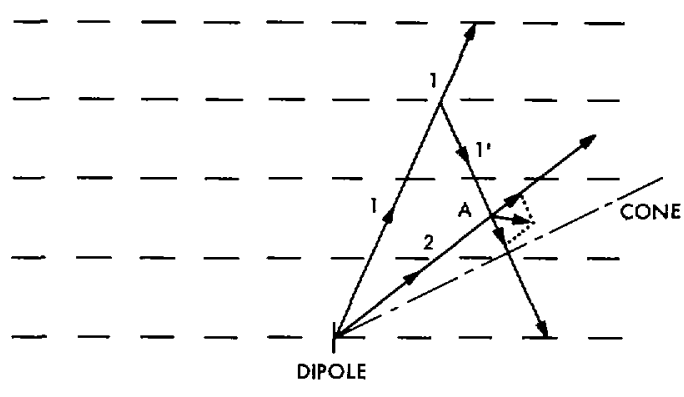

Fig. $9 . \quad \overline{\text { Radiation outside radiation cone. }}$

part of 1 from an inhomogeneity surface), we find a resulting ray outside the phase cone, in the previously forbidden region.

\section{Dipole Radiation in Generally Space-Ttme Periodic Media}

The results found for the sinusoidally space-time periodic media can be extended easily to the generally periodic case.

Let us assume a medium submitted to a propagating periodic disturbance such that

$$
\begin{aligned}
\epsilon=\epsilon(z, t)=\epsilon_{0} \epsilon_{t}\left[1+\epsilon_{1} f(K z-\Omega t)\right], & \text { for a dielectric } \\
N=N(z, t)=N_{0}\left[1+N_{1} f(K z-\Omega t)\right], & \text { for a plasma }
\end{aligned}
$$

where $f(\xi)$ is a normalized periodic function which can be written as

$$
f(\xi)=\sum_{m=-\infty}^{m=+\infty} a_{m} \exp (i m \xi)
$$

Using the same method as for a magnetic dipole in the sinusoidal case we find, instead of (4), (26), (29),

$$
\begin{aligned}
& D_{n} E_{n}+\sum_{l=-\infty}^{l=+\infty} 2 a_{n-l} E_{l}=0, \quad \text { for a dielectric } \\
& D_{n} A_{n}+\sum_{l=-\infty}^{l=+\infty} 2 a_{n-l} A_{l}=0, \quad \text { for a plasma } \\
& D_{n} A_{n z}+\sum_{l} 2 a_{n-l} A_{l z}=0, \quad \text { for a uniaxial plasma }
\end{aligned}
$$

where the $D_{n}$ are given in Table I. These equations can be written in a matrix form

$$
\|M\| \cdot\left[|E| \text { or }|A| \text { or }\left|A_{z}\right|\right]=0
$$

and all the results found in the previous sections are valid here if we use the adequate matrix $\|M\|$.

The same procedure can be followed for the electric dipole case [5], [6].

\section{Conclusion}

The dipole field and radiation pattern in different sinusoidally space-time periodic media were determined and the special effects: caustics, harmonics, radiation even if $\omega<\omega_{p 0}$ in a plasma and radiation outside the cone angle in a uniaxial plasma, were studied. A common formulation was used such that the results are valid for all cases if we use the adequate matrix $\|M\|$. Then our study was generalized to the generally spacetime periodic media.

An interesting extension of this work will be to study the parametric interactions, and their types, which occur between the plane pump wave and the radiated spherical wave.

\section{ACKNOWLEDGMENT}

The author deeply thanks Prof. C. H. Papas for many helpful discussions during this study.

\section{REFERENCES}

[1] J. C. Simon, "Action of a progressive disturbance on a guided electromagnetic wave," IRE Trans. Microwave Theory Tech. vol. MTT-8, pp. 18-29, Jan. 1960.

[2] E. S. Cassedy and A. A. Oliner, "Dispersion relations in timespace periodic media," Proc. IEEE, vol. 51, pp. 1342-1359, Oct. 1963.

[3] B. R. Rao, "Electromagnetic wave propagation in a dielectric medium space-time modulated by a nonlinear pump wave," Proc. IEEE (Lett.), vol. 56, pp. 1630-1631, Sept. 1968 .

[4] J. Askne, "Wave reflection from and propagation in a pumped semi-infinite medium," URSI Symp. Electromagn. Waves, Alta Freq., pp. 216-220, 1968.

[5] C. Elachi, "Electromagnetic wave propagation and source radiation in space-time periodic media." Ph.D. dissertation, California Inst. Technol., Pasadena, 1971.

[6] - "Electromagnetic wave propagation in space-time periodic media," IEEE Trans. Antennas Propagat. (Commun.), vol. AP-20, July 1972 , to be published.

[7] K. F. Casey, "Dipale radiation in a periodically stratified medium," Can. J. Phus., vol. 46, pp. 2543-2551, 1968.

[8] D. B. Singer and T. Tamir-Berman, "Diffraction of light from a filamentary source by parallel acoustic wave fronts," J. Opt. Soc. A mer., vol. 60, pp. 1640-1653, 1970.

[9] K. F. Casey, C. Yeh, and Z. A. Kaprielian, "Cerenkov radiation in inhomogeneous periodic media," Phys. Rev., vol. 140, pp. B768-B775, Nov. 1965.

[10] K. F. Casey and C. Yeh, "Transition radiation in a periodically stratified plasma," Phys. Rev., vol. A-2, pp. 810-818, Sept. 1970. 